\title{
The Cauchy problem for the Gross-Pitaevskii equation
}

\author{
P. Gérard \\ Université Paris-Sud, Mathématiques, Bât. 425, 91405 Orsay cedex, France
}

Received 15 July 2005; accepted 27 September 2005

Available online 2 February 2006

\section{Abstract}

We prove global wellposedness of the two-dimensional and three-dimensional Gross-Pitaevskii equations in the natural energy space.

(c) 2006 L'Association Publications de l'Institut Henri Poincaré. Published by Elsevier B.V. All rights reserved

\section{Résumé}

On établit que le problème de Cauchy pour l'équation de Gross-Pitaevskii est bien posé dans l'espace d'énergie naturel, en dimensions deux et trois.

๑ 2006 L'Association Publications de l'Institut Henri Poincaré. Published by Elsevier B.V. All rights reserved

MSC: 35Q55; 35BXX; 37K05; 37L50; 81Q20

Keywords: Nonlinear Schrödinger equation; Dispersive equations

\section{Introduction}

The Gross-Pitaevskii equation on $\mathbb{R}^{d}$

$$
\mathrm{i} \frac{\partial u}{\partial t}+\Delta u=\left(|u|^{2}-1\right) u
$$

is the Hamiltonian evolution associated to the Ginzburg-Landau energy

$$
\mathcal{E}(u)=\int_{\mathbb{R}^{d}} \frac{1}{2}|\nabla u(x)|^{2}+\frac{1}{4}\left(|u|^{2}-1\right)^{2} \mathrm{~d} x
$$

defined on the space

$$
E=\left\{u \in H_{\mathrm{loc}}^{1}\left(\mathbb{R}^{d}\right): \nabla u \in L^{2}\left(\mathbb{R}^{d}\right),|u|^{2}-1 \in L^{2}\left(\mathbb{R}^{d}\right)\right\} .
$$

Eq. (1.1) arises naturally in several contexts, in particular superfluidity and Bose-Einstein condensates, see $[18,13,5,19]$. The formal Madelung transformation

$$
u=\sqrt{\rho} \mathrm{e}^{\mathrm{i} \phi / 2}
$$

E-mail address: patrick.gerard@math.u-psud.fr (P. Gérard). 
provides a link with a system close to the one describing compressible irrotational fluids, and the question of turbulence effects for this equation has been recently addressed in [17]. On the other hand, existence of progressive waves solutions was recently studied in [2,1,10-12].

It is therefore natural to seek for a theory of the Cauchy problem in the energy space $E$. Notice that, because of the special form of the potential energy, functions in the energy space $E$ do not cancel at infinity, therefore the classical theory of the Cauchy problem for the nonlinear Schrödinger equation developed in $[7,8,15,4]$ does not apply. To our knowledge, the only results of global wellposedness for (1.1) on the whole space $E$ are due to Zhidkov [20,21] and are restricted to one space dimension, without using dispersive properties of the Schrödinger group. However, in an appendix to [2], the strategy of Kato [15] is used to derive global wellposedness in the smaller space $1+H^{1}\left(\mathbb{R}^{d}\right)$ for natural dimensions $d=2,3$.

The purpose of this paper is to show how Strichartz estimates can also be used to obtain global wellposedness of the Cauchy problem for (1.1) in the whole energy space $E$ for $d=2,3$. Before stating our result more precisely, let us introduce a natural structure of metric space on $E$. We recall that Zhidkov introduced in [20] the space

$$
X^{1}(\mathbb{R})=\left\{u \in L^{\infty}(\mathbb{R}): u^{\prime} \in L^{2}(\mathbb{R})\right\}
$$

and proved that the Cauchy problem for (1.1) is well-posed in

$$
\left\{u \in X^{1}(\mathbb{R}):|u|^{2}-1 \in L^{2}(\mathbb{R})\right\} .
$$

In fact, it can be shown that this space coincides with $E$. More generally, we shall show in Section 2 that, in any space dimension $d$,

$$
E \subset X^{1}\left(\mathbb{R}^{d}\right)+H^{1}\left(\mathbb{R}^{d}\right),
$$

where we have set, for every positive integer $k$,

$$
X^{k}\left(\mathbb{R}^{d}\right)=\left\{u \in L^{\infty}\left(\mathbb{R}^{d}\right): \partial^{\alpha} u \in L^{2}\left(\mathbb{R}^{d}\right), 1 \leqslant|\alpha| \leqslant k\right\},
$$

equipped with the natural norm

$$
\|u\|_{X^{k}}=\|u\|_{L^{\infty}}+\sum_{1 \leqslant|\alpha| \leqslant k}\left\|\partial^{\alpha} u\right\|_{L^{2}} .
$$

It is then easy to endow our energy space $E$ with a structure of complete metric space by introducing the following distance function,

$$
d_{E}(u, \tilde{u})=\|u-\tilde{u}\|_{X^{1}+H^{1}}+\left\||u|^{2}-|\tilde{u}|^{2}\right\|_{L^{2}} .
$$

Recall that, given two Banach spaces $X, Y$ of distributions on $\mathbb{R}^{d}$, the Banach norm on the space $X+Y$ is defined by

$$
\|v\|_{X+Y}=\inf \left\{\left\|v_{1}\right\|_{X}+\left\|v_{2}\right\|_{Y}: v=v_{1}+v_{2}, v_{1} \in X, v_{2} \in Y\right\} .
$$

Our result can now be stated as follows.

Theorem 1.1. Let $d \in\{2,3\}$. For every $u_{0} \in E$, there exists a unique solution $u \in C(\mathbb{R}, E)$ of Eq. (1.1) with the initial condition $u(0)=u_{0}$. Moreover, the flow of (1.1) on E enjoys the following additional properties:

- If moreover $\Delta u_{0} \in L^{2}\left(\mathbb{R}^{d}\right)$, then $\Delta u \in C\left(\mathbb{R}, L^{2}\left(\mathbb{R}^{d}\right)\right)$.

- For every time $t, \mathcal{E}(u(t))=\mathcal{E}\left(u_{0}\right)$.

- If $u_{L}(t)=\mathrm{e}^{\mathrm{i} t \Delta} u_{0}$ denotes the solution of the linear Schrödinger equation with the same Cauchy data, $u-u_{L} \in$ $C\left(\mathbb{R}, H^{1}\left(\mathbb{R}^{d}\right)\right)$.

- For every $R>0$, for every $T>0$, there exists $C>0$ such that, for every $u_{0}, \tilde{u}_{0} \in E$ such that $\mathcal{E}\left(u_{0}\right) \leqslant R$ and $\mathcal{E}\left(\tilde{u}_{0}\right) \leqslant R$, the corresponding solutions $u, \tilde{u}$ satisfy

$$
\sup _{|t| \leqslant T} d_{E}(u(t), \tilde{u}(t)) \leqslant C d_{E}\left(u_{0}, \tilde{u}_{0}\right) .
$$


Let us mention that local existence results in the spaces $X^{k}\left(\mathbb{R}^{d}\right), k>d / 2$, were already proved by Gallo [6], and that a global existence result in $X^{2}\left(\mathbb{R}^{2}\right)$ has been obtained recently by Goubet [9] using logarithmic estimates as in Brezis-Gallouët 's contribution to cubic nonlinear Schrödinger equation on plane domains [3].

In the special case $d=3$, it is in fact possible to describe the energy space in a simpler way. Denote by

$$
\dot{H}^{1}\left(\mathbb{R}^{3}\right)=\mathcal{D}^{1,2}\left(\mathbb{R}^{3}\right)=\left\{v \in L^{6}\left(\mathbb{R}^{3}\right): \nabla v \in L^{2}\left(\mathbb{R}^{3}\right)\right\}
$$

the completion of the space $C_{0}^{\infty}\left(\mathbb{R}^{3}\right)$ for the Dirichlet norm $\|\nabla v\|_{L^{2}}$. Then one can prove that

$$
E=\left\{u=c+v: c \in \mathbb{C},|c|=1, v \in \dot{H}^{1}\left(\mathbb{R}^{3}\right),|v|^{2}+2 \operatorname{Re}\left(c^{-1} v\right) \in L^{2}\left(\mathbb{R}^{3}\right)\right\} .
$$

Moreover, the distance function $d_{E}$ is equivalent to

$$
\delta_{E}(c+v, \tilde{c}+\tilde{v})=|c-\tilde{c}|+\|\nabla v-\nabla \tilde{v}\|_{L^{2}}+\left\||v|^{2}+2 \operatorname{Re}\left(c^{-1} v\right)-|\tilde{v}|^{2}-2 \operatorname{Re}\left(\tilde{c}^{-1} \tilde{v}\right)\right\|_{L^{2}}
$$

and the dynamics of (1.1) keep $c$ invariant. No such simple description is available in two space dimensions, since elements of $E$ may have complicated oscillations at infinity, for instance

$$
u(x)=\mathrm{e}^{\mathrm{i}(\log (2+|x|))^{\alpha}}, \quad \alpha<\frac{1}{2} .
$$

This paper is organized as follows. In Section 2, we prove the inclusion $E \subset X^{1}+H^{1}$ and we establish that the linear Schrödinger group acts nicely on the nonlinear energy space $E$ if $d=2$, 3. Section 3 is devoted to the proof of Theorem 1.1 by a contraction argument. An important point consists in observing that the nonlinear drift $w=u-u_{L}$ belongs to $C\left(\mathbb{R}, H^{1}\left(\mathbb{R}^{d}\right)\right)$. Finally, in Section 4 we prove the above-mentioned description of the dynamics on the space $E$ in the special case $d=3$. We also discuss the role of the dimension in studying how the energy functional measures the distance to the circle of constants of null energy. Finally, we sketch a generalization of Theorem 1.1 to small energy solutions in four space dimensions.

\section{The energy space and the linear Schrödinger equation}

In this section, we prove some preliminary results concerning the structure of the energy space $E$ and the action of the Schrödinger group on $E$.

\subsection{The energy space, Sobolev spaces and Zhidkov spaces}

Recall that the energy space $E$ is defined in (1.3) and that the Zhidkov space $X^{1}\left(\mathbb{R}^{d}\right)$ is defined in (1.4). Our first result is the following observation.

Lemma 1. In any space dimension $d$, we have

$$
E \subset X^{1}\left(\mathbb{R}^{d}\right)+H^{1}\left(\mathbb{R}^{d}\right)
$$

and there exists $C>0$ such that, for every $u \in E$,

$$
\|u\|_{X^{1}+H^{1}} \leqslant C(1+\sqrt{\mathcal{E}(u)}) .
$$

Proof. Let $\chi \in C_{0}^{\infty}(\mathbb{C})$ such that $0 \leqslant \chi \leqslant 1, \chi(z)=1$ for $|z| \leqslant 2$, and $\chi(z)=0$ for $|z| \geqslant 3$. Let us decompose

$$
u=u_{1}+u_{2}, \quad u_{1}=\chi(u) u, \quad u_{2}=(1-\chi(u)) u .
$$

We have

$$
\left\|u_{1}\right\|_{L^{\infty}} \leqslant 3
$$

and, since $|u| \geqslant 2$ on the support of $u_{2}$,

$$
\left|u_{2}\right| \leqslant\left.|| u\right|^{2}-1 \mid
$$

thus

$$
\left\|u_{2}\right\|_{L^{2}} \leqslant\left\||u|^{2}-1\right\|_{L^{2}} \leqslant 2 \sqrt{\mathcal{E}(u)}
$$


On the other hand,

$$
\nabla u_{1}=(\chi(u)+u \partial \chi(u)) \nabla u+u \bar{\partial} \chi(u) \nabla \bar{u} ; \quad \nabla u_{2}=(1-\chi(u)-u \partial \chi(u)) \nabla u-u \bar{\partial} \chi(u) \nabla \bar{u} .
$$

This implies easily, for some $A=A(\chi)$,

$$
\left\|\nabla u_{1}\right\|_{L^{2}}+\left\|\nabla u_{2}\right\|_{L^{2}} \leqslant A\|\nabla u\|_{L^{2}} \leqslant \sqrt{2} A \sqrt{\mathcal{E}(u)} .
$$

Hence

$$
\left\|u_{1}\right\|_{X^{1}}+\left\|u_{2}\right\|_{H^{1}} \leqslant 3+(2+\sqrt{2} A) \sqrt{\mathcal{E}(u)} .
$$

The proof is complete.

Remark 2.1. Notice that, in the special case of dimension $d=1$, the above lemma reads $E \subset X^{1}(\mathbb{R})$.

We close this subsection by an elementary lemma which will be useful in the sequel.

Lemma 2. If $d=2,3,4$, we have $E+H^{1} \subset E$ and, for every $v \in E, w \in H^{1}$,

$$
\left\||v+w|^{2}-1\right\|_{L^{2}} \leqslant\left\||v|^{2}-1\right\|_{L^{2}}+C(1+\sqrt{\mathcal{E}(v)})\left(\|w\|_{L^{2}}+\|w\|_{L^{4}}\right)+\|w\|_{L^{4}}^{2} .
$$

Moreover, for every $v \in E, \tilde{v} \in E$ and $w \in H^{1}, \tilde{w} \in H^{1}$,

$$
\begin{aligned}
d_{E}(v+w, \tilde{v}+\tilde{w}) \leqslant & C\left(1+\|w\|_{H^{1}}+\|\widetilde{w}\|_{H^{1}}\right) d_{E}(v, \tilde{v}) \\
& +C\left(1+\sqrt{\mathcal{E}(v)}+\sqrt{\mathcal{E}(\tilde{v})}+\|w\|_{H^{1}}+\|\widetilde{w}\|_{H^{1}}\right)\|w-\widetilde{w}\|_{H^{1}} .
\end{aligned}
$$

Proof. Given $v \in E, w \in H^{1}$, trivially $\nabla(v+w) \in L^{2}$. Then we expand

$$
|v+w|^{2}-1=|v|^{2}-1+2 \operatorname{Re}(\bar{v} w)+|w|^{2}
$$

and this quantity belongs to $L^{2}$ since $v \in L^{\infty}+L^{4}$ and $w \in L^{2} \cap L^{4}$ by Lemma 1 and the Sobolev inequality. This gives estimate (2.2). Moreover,

$$
\|v+w-\tilde{v}-\tilde{w}\|_{X^{1}+H^{1}} \leqslant\|v-\tilde{v}\|_{X^{1}+H^{1}}+\|w-\widetilde{w}\|_{H^{1}} .
$$

Finally, the estimate of $\left\||v+w|^{2}-|\tilde{v}+\widetilde{w}|^{2}\right\|_{L^{2}}$ can be established along the same lines as (2.2).

\subsection{Action of the linear Schrödinger group on the energy space}

We start with a general statement about the spaces $X^{k}+H^{k}$. Denote by $S(t)=\mathrm{e}^{\mathrm{i} t \Delta}$ the linear Schrödinger propagator.

Lemma 3. Let $d$ be a positive integer. For every positive integer $k$, for every $t \in \mathbb{R}, S(t)$ maps $X^{k}\left(\mathbb{R}^{d}\right)+H^{k}\left(\mathbb{R}^{d}\right)$ into itself, with the estimates

$$
\|S(t) f\|_{X^{k}+H^{k}} \leqslant C(1+|t|)^{1 / 2}\|f\|_{X^{k}+H^{k}},
$$

and

$$
\|S(t) f-f\|_{L^{2}} \leqslant C|t|^{1 / 2}\|\nabla f\|_{L^{2}} .
$$

Moreover, if $f \in X^{k}\left(\mathbb{R}^{d}\right)+H^{k}\left(\mathbb{R}^{d}\right)$, the map

$$
t \in \mathbb{R} \mapsto S(t) f \in X^{k}\left(\mathbb{R}^{d}\right)+H^{k}\left(\mathbb{R}^{d}\right)
$$

is continuous. 
Proof. Since $S(t)$ acts isometrically on $H^{k}\left(\mathbb{R}^{d}\right)$, it is enough to assume $f \in X^{k}\left(\mathbb{R}^{d}\right)$. We just write

$$
S(t) f=f+S(t) f-f
$$

and we are led to prove that $S(t) f-f \in H^{k}\left(\mathbb{R}^{d}\right)$ with

$$
\|S(t) f-f\|_{H^{k}} \leqslant C(1+|t|)^{1 / 2}\|f\|_{X^{k}} \quad \text { and } \quad\|S(t) f-f\|_{H^{k}} \rightarrow 0
$$

as $t$ tends to 0 . For $1 \leqslant|\alpha| \leqslant k$, it is clear that $\partial^{\alpha}(S(t) f-f)=(S(t)-1) \partial^{\alpha} f \in L^{2}\left(\mathbb{R}^{d}\right)$ with

$$
\left\|\partial^{\alpha}(S(t) f-f)\right\|_{L^{2}} \leqslant C\left\|\partial^{\alpha} f\right\|_{L^{2}} \text { and }\left\|\partial^{\alpha}(S(t) f-f)\right\|_{L^{2}} \rightarrow 0
$$

as $t$ tends to 0 . Moreover, introducing $\chi \in C_{0}^{\infty}(\mathbb{R})$ such that $\chi=1$ near the origin, we can write

$$
\mathrm{e}^{-\mathrm{i} t|\xi|^{2}}-1=\sum_{j=1}^{d} g_{j}(t, \xi) \xi_{j}
$$

with

$$
g_{j}(t, \xi)=-\mathrm{i} t \chi\left(t|\xi|^{2}\right) \xi_{j} \int_{0}^{1} \mathrm{e}^{-\mathrm{i} s t|\xi|^{2}} \mathrm{~d} s+\frac{1-\chi\left(t|\xi|^{2}\right)}{|\xi|^{2}} \xi_{j}\left(\mathrm{e}^{-\mathrm{i} t|\xi|^{2}}-1\right)=\mathcal{O}\left(|t|^{1 / 2}\right) .
$$

Consequently,

$$
S(t) f-f=\mathrm{i} \sum_{j=1}^{d} g_{j}(t, D) \partial_{j} f \in L^{2}\left(\mathbb{R}^{d}\right)
$$

and this yields (2.3). The last assertion is a simple consequence of (2.3) and of the continuity of $t \mapsto S(t) f \in L^{2}$ if $f \in L^{2}$.

Remark 2.2. In the case $k>d / 2$, we have $H^{k}\left(\mathbb{R}^{d}\right) \subset X^{k}\left(\mathbb{R}^{d}\right)$ and we recover the fact that $S(t)$ acts on $X^{k}\left(\mathbb{R}^{d}\right)$ (see Gallo [6] for the original proof).

Combining the previous lemmas, it is now possible to show that the energy space is kept invariant by the Schrödinger group if the dimension is not larger than 4 .

Proposition 2.3. Let $d \in\{2,3,4\}$. For every $t \in \mathbb{R}, S(t)(E) \subset E$ and, for every $u_{0} \in E$, the map

$$
t \in \mathbb{R} \mapsto S(t) u_{0} \in E
$$

is continuous. Moreover, for every $R>0$, for every $T>0$, there exists $C>0$ such that, for every $u_{0}, \tilde{u}_{0} \in E$ such that $\mathcal{E}\left(u_{0}\right) \leqslant R$ and $\mathcal{E}\left(\tilde{u}_{0}\right) \leqslant R$,

$$
\sup _{|t| \leqslant T} d_{E}\left(S(t) u_{0}, S(t) \tilde{u}_{0}\right) \leqslant C d_{E}\left(u_{0}, \tilde{u}_{0}\right) .
$$

Finally, if $d=2,3$, for every $R>0$ there exists $T(R)>0$ such that, for every $u_{0} \in E$ such that $\mathcal{E}\left(u_{0}\right) \leqslant R$, we have

$$
\sup _{|t| \leqslant T(R)} \mathcal{E}\left(S(t) u_{0}\right) \leqslant 2 R .
$$

If $d=4$, there exists $A>0$ such that, for every $u_{0} \in E$ such that $\mathcal{E}\left(u_{0}\right) \leqslant 1$, we have

$$
\sup _{|t| \leqslant 1} \mathcal{E}\left(S(t) u_{0}\right) \leqslant A \mathcal{E}\left(u_{0}\right)
$$

Proof. Writing again

$$
S(t) u_{0}=u_{0}+\left(S(t) u_{0}-u_{0}\right),
$$


and using Lemmas 2 and 3, we have easily $S(t)(E) \subset E$, while the continuity property

$$
S(t) u_{0} \underset{t \rightarrow 0}{\longrightarrow} u_{0} \text { in } E
$$

is a consequence of the second estimate in Lemma 2 and of Lemma 3. Finally, estimates (2.5) and (2.6) are consequences of (2.2). Indeed, if $d=2,3$, the combination of (2.3) and of the interpolation inequality

$$
\|w\|_{L^{4}} \leqslant C\|w\|_{L^{2}}^{\theta}\|\nabla w\|_{L^{2}}^{1-\theta},
$$

for some $\theta \in] 0,1$ [, allows to make the $L^{4}$ norm of $S(t) u_{0}-u_{0}$ uniformly small for $\mathcal{E}\left(u_{0}\right) \leqslant R$ and $|t| \leqslant T(R)$. If $d=4,4$ is exactly the Sobolev exponent and we must be content with the linear estimate (2.6).

\section{Proof of the main theorem}

In this section, we prove Theorem 1.1, thus $d \in\{2,3\}$. We start by observing that the nonlinear drift belongs to $H^{1}\left(\mathbb{R}^{d}\right)$ at every time. Then we solve the equation by a contraction argument in the space $C([-T, T], E)$ for $T$ small enough with respect to the energy of the data. Finally we establish propagation of the regularity of the solution and conservation of the energy, which leads classically to global existence of the solution.

\subsection{Estimates on the nonlinear drift}

Let $u_{0} \in E$. As in the previous section, we denote by $u_{L}$ the solution of the linear Schrödinger equation such that $u_{L}(0)=u_{0}$. Then Eq. (1.1) is, at least formally, equivalent to

$$
u(t)=u_{L}(t)-\mathrm{i} \int_{0}^{t} S(t-\tau)\left[\left(|u|^{2}-1\right) u(\tau)\right] \mathrm{d} \tau .
$$

Therefore it is natural to study first the map

$$
u \mapsto F(u)=\left(|u|^{2}-1\right) u
$$

for $u \in E$. The following lemma is an elementary consequence of Lemma 1 and of the Sobolev inequalities.

Lemma 4. Assume $d \in\{2,3\}$. Then, for every $u \in E, F(u) \in L^{2}+L^{3 / 2}$ and $\nabla(F(u)) \in L^{2}+L^{6 / 5}$. Moreover, for every $R>0$, there exists $C(R)$ such that, for every $u$, $\tilde{u}$ in $E$ such that $\mathcal{E}(u) \leqslant R, \mathcal{E}(\tilde{u}) \leqslant R$, we have

$$
\|F(u)-F(\tilde{u})\|_{L^{2}+L^{3 / 2}}+\|\nabla(F(u))-\nabla(F(\tilde{u}))\|_{L^{2}+L^{6 / 5}} \leqslant C(R) d_{E}(u, \tilde{u}) .
$$

Proof. Since $u \in X^{1}+H^{1}$ and $d \leqslant 3$, we have $u \in L^{\infty}+L^{6}$. Since $|u|^{2}-1 \in L^{2}$, Hölder's inequality then implies $F(u) \in L^{2}+L^{3 / 2}$. Moreover,

$$
F(u)-F(\tilde{u})=\left(|u|^{2}-|\tilde{u}|^{2}\right) u+(u-\tilde{u})\left(|\tilde{u}|^{2}-1\right),
$$

therefore

$$
\|F(u)-F(\tilde{u})\|_{L^{2}+L^{3 / 2}} \leqslant\left\||u|^{2}-|\tilde{u}|^{2}\right\|_{L^{2}}\|u\|_{L^{\infty}+L^{6}}+\left\||\tilde{u}|^{2}-1\right\|_{L^{2}}\|u-\tilde{u}\|_{L^{\infty}+L^{6}} \leqslant C(R) d_{E}(u, \tilde{u}) .
$$

As for the gradient, we have

$$
\nabla(F(u))=\left(2|u|^{2}-1\right) \nabla u+u^{2} \nabla \bar{u} .
$$

Using that $2|u|^{2}-1$ and $u^{2}$ belong to $L^{\infty}+L^{3}$, and that $\nabla u \in L^{2}$, we conclude $\nabla(F(u)) \in L^{2}+L^{6 / 5}$. Moreover,

$$
\begin{aligned}
\nabla(F(u))-\nabla(F(\tilde{u}))= & 2(u-\tilde{u}) \bar{u} \nabla u+2 \tilde{u} \overline{(u-\tilde{u})} \nabla u+\left(2|\tilde{u}|^{2}-1\right)(\nabla u-\nabla \tilde{u}) \\
& +(u-\tilde{u})(u+\tilde{u}) \nabla u+\tilde{u}^{2}(\nabla u-\nabla \tilde{u}) .
\end{aligned}
$$

This yields 


$$
\begin{aligned}
\|\nabla(F(u))-\nabla(F(\tilde{u}))\|_{L^{2}+L^{6 / 5}} \leqslant & C\left(\|u\|_{L^{\infty}+L^{6}}+\|\tilde{u}\|_{L^{\infty}+L^{6}}\right)\|\nabla u\|_{L^{2}}\|u-\tilde{u}\|_{L^{\infty}+L^{6}} \\
& +C\left(1+\|\tilde{u}\|_{L^{\infty}+L^{6}}^{2}\|\nabla u-\nabla \tilde{u}\|_{L^{2}}\right.
\end{aligned}
$$

and (3.1) follows.

Next we recall the nonhomogeneous generalized Strichartz estimates $[8,16]$. We denote by $r^{\prime}$ the conjugate exponent of $r$. We shall say that a pair $(p, q)$ of positive numbers is admissible if

$$
\frac{2}{p}+\frac{d}{q}=\frac{d}{2}, \quad p \geqslant 2,(p, q) \neq(2, \infty) .
$$

Notice that $q<\infty$ can be as large as we want if $d=2$, while $q$ is limited by the Sobolev exponent $2^{*}=(2 d) /(d-2)$ if $d \geqslant 3$. For every admissible pair $\left(p_{1}, q_{1}\right)$, for every $T>0$, for every $f \in L^{p_{1}^{\prime}}\left([-T, T], L^{q_{1}^{\prime}}\left(\mathbb{R}^{d}\right)\right)$, the function

$$
w(t)=\int_{0}^{t} S(t-\tau) f(\tau) \mathrm{d} \tau
$$

satisfies $w \in L^{p_{2}}\left([-T, T], L^{q_{2}}\left(\mathbb{R}^{d}\right)\right)$ for every admissible pair $\left(p_{2}, q_{2}\right)$, with the estimate

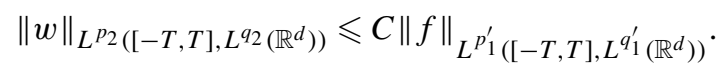

Combining these estimates with Lemma 4 , we infer

Lemma 5. Let $d \in\{2,3\}$. For every $u \in C([-T, T], E)$, the quantity

$$
\Phi(u)(t)=-\mathrm{i} \int_{0}^{t} S(t-\tau)[F(u(\tau))] \mathrm{d} \tau
$$

belongs to $C\left([-T, T], H^{1}\left(\mathbb{R}^{d}\right)\right)$. Moreover, if $T \leqslant 1$, for every $R>0$ there exists $C(R)>0$ such that, if $u$, $\tilde{u}$ satisfy

$$
\forall t \in[-T, T], \quad \mathcal{E}(u(t)) \leqslant R, \quad \mathcal{E}(\tilde{u}(t)) \leqslant R,
$$

then, if $T \leqslant 1$,

$$
\sup _{|t| \leqslant T}\|\Phi(u)(t)-\Phi(\tilde{u})(t)\|_{H^{1}} \leqslant C(R) T^{1 / 2} \sup _{|t| \leqslant T} d_{E}(u(t), \tilde{u}(t)) .
$$

Proof. From Lemma 4, we know that $F(u) \in L^{\infty}\left([-T, T], L^{2}+L^{3 / 2}\right)$, hence, applying (3.3) with $q_{2}=2$ and $q_{1}=2$ or $q_{1}=3$, we obtain $\Phi(u) \in C\left([-T, T], L^{2}\left(\mathbb{R}^{d}\right)\right)$. Observing that

$$
\nabla \Phi(u)(t)=-\mathrm{i} \int_{0}^{t} S(t-\tau)[\nabla(F(u(\tau)))] \mathrm{d} \tau
$$

we argue similarly with $q_{1}=2$ or $q_{1}=6$, and this gives $\nabla(\Phi(u)) \in C\left([-T, T], L^{2}\left(\mathbb{R}^{d}\right)\right)$. Estimate (3.5) similarly follows from (3.1), the factor $T^{1 / 2}$ coming from the fact that $p_{1}^{\prime} \leqslant 2$ in estimates (3.3) and that $T \leqslant 1$.

\subsection{Local wellposedness}

As a first classical step, we shall solve the Cauchy problem for (1.1) locally in time. Denote by $u_{L}$ the solution of the linear Schrödinger equation,

$$
u_{L}(t)=S(t) u_{0}
$$

The Duhamel equation for $u$ reads

$$
u(t)=u_{L}(t)+\Phi(u)(t),
$$

where $\Phi$ was defined in (3.4). 
Proposition 3.1. Let $d=2$, 3. For every $R>0$, there exists $T>0$ such that, for every $u_{0} \in E$ with $\mathcal{E}\left(u_{0}\right) \leqslant R$, there exists a unique solution

$$
u \in C([-T, T], E)
$$

of Eq. (1.1) with $u(0)=u_{0}$. Moreover, if $\tilde{u}_{0} \in E$ satisfies $\mathcal{E}\left(\tilde{u}_{0}\right) \leqslant R$ and $\tilde{u}$ is the corresponding solution of (1.1), then we have the Lipschitz estimate,

$$
\sup _{|t| \leqslant T} d_{E}(u(t), \tilde{u}(t)) \leqslant C(R) d_{E}\left(u_{0}, \tilde{u}_{0}\right) .
$$

Proof. Denote by $X_{T}$ the complete metric space of functions $u \in C([-T, T], E)$ such that $\sup _{|t| \leqslant T} \mathcal{E}(u(t)) \leqslant 3 R$. We shall prove that, for $T$ small enough, the map

$$
u \mapsto u_{L}+\Phi(u)
$$

is a contraction of $X_{T}$. Given $u_{0} \in E$ such that $\mathcal{E}\left(u_{0}\right) \leqslant R$, Proposition 2.3 implies that, for $T$ small enough with respect to $R$,

$$
\sup _{|t| \leqslant T} \mathcal{E}\left(u_{L}(t)\right) \leqslant 2 R
$$

On the other hand, applying estimate (3.5) in Lemma 5 in the special case where $\tilde{u}$ is a constant of modulus 1 ,

$$
\sup _{|t| \leqslant T}\|\Phi(u)(t)\|_{H^{1}} \leqslant C(R) T^{1 / 2} .
$$

It remains to apply Lemma 2 to conclude, for $T$ small enough with respect to $R$,

$$
\sup _{|t| \leqslant T} \mathcal{E}\left(u_{L}(t)+\Phi(u)(t)\right) \leqslant 3 R,
$$

hence $u \mapsto u_{L}+\Phi(u)$ acts on $X_{T}$. Then we combine the second estimate of Lemma 2 with estimate (3.5) of Lemma 5 to obtain, if $u, \tilde{u} \in X_{T}$,

$$
\begin{aligned}
\sup _{|t| \leqslant T} d_{E}\left(u_{L}(t)+\Phi(u(t)), u_{L}(t)+\Phi(\tilde{u}(t))\right) & \leqslant C(R) \sup _{|t| \leqslant T}\|\Phi(u)(t)-\Phi(\tilde{u}(t))\|_{H^{1}} \\
& \leqslant C^{\prime}(R) T^{1 / 2} \sup _{|t| \leqslant T} d_{E}(u(t), \tilde{u}(t)) .
\end{aligned}
$$

Choosing $T$ small enough with respect to $R$ completes the proof of the contraction argument. The proof of the Lipschitz estimate is similar.

Remark 3.2. An equivalent approach would consist in solving the equation

$$
w=\Phi\left(u_{L}+w\right)
$$

in the space $C\left([0, T], H^{1}\left(\mathbb{R}^{d}\right)\right)$ for $T=T(R)$ small enough.

\subsection{Regularity and conservation of the energy}

We now come to the regularity of the solution constructed in the previous subsection.

Assume that $\Delta u_{0} \in L^{2}\left(\mathbb{R}^{d}\right), d=2,3$. Then

$$
u_{0} \in X^{2}+H^{2}=X^{2}
$$

by taking into account that $d \leqslant 3$. Therefore it is classical that the map

$$
u \mapsto\left(|u|^{2}-1\right) u
$$

leaves $X^{2}$ invariant, and is Lipschitz continuous on the bounded subsets of $X^{2}$. Consequently, Eq. (1.1) has a maximal solution belonging to $C(]-T_{*}, T^{*}\left[, X^{2}\right)$, which blows up in $X^{2}$ as $t$ approaches a boundary point $-T_{*}$ or $T^{*}$ if this point is finite. Let us show that $]-T_{*}, T^{*}\left[=\mathbb{R}\right.$. As a first step, we shall prove that, for every $R>0$, there exits $T_{1}>0$ 
depending only on $R$ such that, if $\mathcal{E}\left(u_{0}\right) \leqslant R$ and $\Delta u_{0} \in L^{2}$, then $\left.\left[-T_{1}, T_{1}\right] \subset\right]-T_{*}, T^{*}$ [. Denote by $T$ the time constructed in Proposition 3.1, so that $u(t)$ stays bounded in $E$ for $|t| \leqslant T$ by a constant depending only on $R$. Then, for $t \in[-T, T] \cap]-T_{*}, T^{*}[$, we have

$$
\left|\Delta\left(\left(|u|^{2}-1\right) u\right)\right| \leqslant\left(3|u|^{2}+1\right)|\Delta u|+2|u||\nabla u|^{2}
$$

so that, for every $T^{\prime}$ such that $\left.\left[-T^{\prime}, T^{\prime}\right] \subset[-T, T] \cap\right]-T_{*}, T^{*}[$,

$$
\left\|\Delta\left(\left(|u|^{2}-1\right) u\right)\right\|_{L^{\infty}\left(\left[-T^{\prime}, T^{\prime}\right], L^{2}+L^{6 / 5}\right)} \leqslant C(R)\|\Delta u\|_{L^{\infty}\left(\left[-T^{\prime}, T^{\prime}\right], L^{2}\right)} .
$$

By estimate (3.3), we conclude

$$
\|\Delta u\|_{L^{\infty}\left(\left[-T^{\prime}, T^{\prime}\right], L^{2}\right)} \leqslant C\left\|\Delta u_{0}\right\|_{L^{2}}+C(R) \sqrt{T^{\prime}}\|\Delta u\|_{L^{\infty}\left(\left[-T^{\prime}, T^{\prime}\right], L^{2}\right)}
$$

hence $u$ stays bounded in $X^{2}$ for $|t| \leqslant T_{1}(R)$ small enough, and thus

$$
\left.\left[-T_{1}(R), T_{1}(R)\right] \subset\right]-T_{*}, T^{*}[
$$

as claimed.

Since $\partial_{t} u \in L^{\infty}\left(\left[-T_{1}, T_{1}\right], L^{2}\right)$, we can compute, using Eq. (1.1),

$$
\frac{\mathrm{d}}{\mathrm{d} t} \mathcal{E}(u(t))=\int_{\mathbb{R}^{d}}\left(\operatorname{Re}\left(\nabla \bar{u} \cdot \nabla \partial_{t} u\right)+\left(|u|^{2}-1\right) \operatorname{Re}\left(\bar{u} \partial_{t} u\right)\right) \mathrm{d} x=0 .
$$

Consequently we have $\mathcal{E}(u(t))=\mathcal{E}\left(u_{0}\right) \leqslant R$ on $\left[-T_{1}, T_{1}\right]$, and, by iterating this argument and Proposition 3.1, we conclude that $u$ can be extended as a global solution with $u \in C\left(\mathbb{R}, X^{2}\right)$ and $\mathcal{E}(u(t))=\mathcal{E}\left(u_{0}\right)$ for every $t \in \mathbb{R}$.

Let us come back to the general case $u_{0} \in E$, and let us prove global existence and conservation of energy in this case. By iterating Proposition 3.1, it is enough to show that the energy is constant on the time interval $[-T, T]$. In view of conservation of energy for regular solutions, it is enough to prove that every data $u_{0} \in E$ can be approximated in the sense of $d_{E}$ by a sequence $u_{0}^{\varepsilon}$ of elements of $E$ such that $\Delta u_{0}^{\varepsilon} \in L^{2}$. Indeed, by Proposition 3.1, we shall have

$$
\sup _{|t| \leqslant T} d_{E}\left(u^{\varepsilon}(t), u(t)\right) \rightarrow 0
$$

therefore, for every $t$,

$$
\mathcal{E}\left(u^{\varepsilon}(t)\right) \rightarrow \mathcal{E}(u(t))
$$

and conservation of energy for $u^{\varepsilon}$ will imply conservation of energy for $u$. Therefore the proof of Theorem 1.1 is completed by the following lemma.

Lemma 6. Let $d=2$, 3. For every $u \in E$, define $u^{\varepsilon}=\rho^{\varepsilon} * u$, where

$$
\rho^{\varepsilon}(x)=\varepsilon^{-d} \rho\left(\frac{x}{\varepsilon}\right)
$$

is a classical mollifier on $\mathbb{R}^{d}$, with $\rho \in C_{0}^{\infty}$. Then $d_{E}\left(u^{\varepsilon}, u\right) \rightarrow 0$ as $\varepsilon$ tends to 0 .

Proof. We have to show that $u^{\varepsilon}$ converges to $u$ in $X^{1}+H^{1}$, and that $\left|u^{\varepsilon}\right|^{2}-1$ converges to $|u|^{2}-1$ in $L^{2}$. Writing

$$
u=\chi(D) u_{1}+(1-\chi(D)) u_{1}+u_{2}, \quad u_{1} \in X^{1}, u_{2} \in H^{1},
$$

with $\chi \in C_{0}^{\infty}\left(\mathbb{R}^{d}\right)$ satisfying $\chi=1$ near the origin, we have

$$
u^{\varepsilon}=\hat{\rho}(\varepsilon D) \chi(D) u_{1}+\rho^{\varepsilon} *\left[(1-\chi(D)) u_{1}+u_{2}\right],
$$

and the first term in the right-hand side converges to $\chi(D) u_{1}$ in $X^{1}$, while the second one converges to $(1-\chi(D)) u_{1}+$ $u_{2}$ in $H^{1}$. This proves the first convergence. The second one is a little more tricky. We write

$$
\begin{aligned}
\left|u^{\varepsilon}(x)\right|^{2}-1 & =\varepsilon^{-2 d} \iint_{\mathbb{R}^{d} \times \mathbb{R}^{d}} \rho\left(\frac{x-y}{\varepsilon}\right) \rho\left(\frac{x-y^{\prime}}{\varepsilon}\right)\left[u(y) \bar{u}\left(y^{\prime}\right)-1\right] \mathrm{d} y \mathrm{~d} y^{\prime} \\
& =\rho^{\varepsilon} *\left(|u|^{2}-1\right)(x)+r^{\varepsilon}(x),
\end{aligned}
$$


with

$$
r^{\varepsilon}(x)=\int_{0}^{1} \varepsilon^{-2 d} \int_{\mathbb{R}^{d} \times \mathbb{R}^{d}} \rho\left(\frac{x-y}{\varepsilon}\right) \rho\left(\frac{x-y^{\prime}}{\varepsilon}\right) u(y)\left(y-y^{\prime}\right) \cdot \nabla \bar{u}\left(y+\theta\left(y^{\prime}-y\right)\right) \mathrm{d} y \mathrm{~d} y^{\prime} \mathrm{d} \theta .
$$

Notice that $\left|y^{\prime}-y\right| \lesssim \varepsilon$ in the above integral, therefore we set $y^{\prime}=y+\varepsilon a$ and we obtain

$$
\left|r^{\varepsilon}(x)\right| \leqslant C \varepsilon \int_{0}^{1} \mathrm{~d} \theta \int_{|a| \lesssim 1} \mathrm{~d} a\left(\int_{\mathbb{R}^{d}} \varepsilon^{-d} \rho_{a}\left(\frac{x-y}{\varepsilon}\right)|u(y)||\nabla u(y+\varepsilon \theta a)| \mathrm{d} y\right),
$$

with $\rho_{a}(z)=\rho(z) \rho(z-a)$. Since $u \in L^{\infty}+L^{6}$ and $\nabla u \in L^{2}$, the function

$$
y \mapsto|u(y)||\nabla u(y+\varepsilon \theta a)|
$$

has a uniform bound in $L^{2}+L^{3 / 2}$ as $\varepsilon, \theta, a$ vary. Consequently, by the Young inequality,

$$
\left\|r^{\varepsilon}\right\|_{L^{2}} \leqslant C \varepsilon \varepsilon^{-d / 6} \rightarrow 0,
$$

which completes the proof.

\section{The three-dimensional case}

In this section, we show that the description of the energy space and of the dynamics of (1.1) can be simplified in the case $d=3$.

\subsection{On the structure of the energy space}

For every complex number of modulus 1 , we introduce

$$
F_{c}=\left\{v \in \dot{H}^{1}\left(\mathbb{R}^{3}\right):|v|^{2}+2 \operatorname{Re}\left(c^{-1} v\right) \in L^{2}\left(\mathbb{R}^{3}\right)\right\},
$$

and, for $v \in F_{c}, \tilde{v} \in F_{\tilde{c}}$, we set

$$
\delta_{c, \tilde{c}}(v, \tilde{v})=\|\nabla v-\nabla \tilde{v}\|_{L^{2}}+\left\||v|^{2}+2 \operatorname{Re}\left(c^{-1} v\right)-|\tilde{v}|^{2}-2 \operatorname{Re}\left(\tilde{c}^{-1} \tilde{v}\right)\right\|_{L^{2}} .
$$

In particular, $\delta_{c, c}$ is a distance function on $F_{c}$.

Proposition 4.1. The space $E$ is exactly the set of functions of the form

$$
u=c+v
$$

where $c$ is a complex number of modulus 1 and $v \in F_{c}$. Moreover, the distance function $d_{E}$ given by (1.5) is equivalent to

$$
\delta_{E}(c+v, \tilde{c}+\tilde{v})=|c-\tilde{c}|+\delta_{c, \tilde{c}}(v, \tilde{v}) .
$$

Proof. Since $|c+v|^{2}-1=|v|^{2}+2 \operatorname{Re}\left(c^{-1} v\right)$, it is trivial that $c+v \in E$ for any $c$ of modulus 1 , for any $v \in F_{c}$. The converse will be a consequence of the following lemma.

Lemma 7. Let $u \in H_{\mathrm{loc}}^{1}\left(\mathbb{R}^{3}\right)$ such that $\nabla u \in L^{2}\left(\mathbb{R}^{3}\right)$. Then the family $U_{r}$ of functions defined on the unit sphere $S^{2}$ by

$$
U_{r}(\omega)=u(r \omega)
$$

converges in $L^{2}\left(S^{2}\right)$ to a constant $c(u)$ as $r$ goes to infinity. Moreover $u$ belongs to $\dot{H}^{1}\left(\mathbb{R}^{3}\right)$ if and only if $c(u)=0$.

Proof. The assumption $\nabla u \in L^{2}\left(\mathbb{R}^{3}\right)$ reads

$$
\int_{0}^{\infty} r^{2}\left\|\partial_{r} U_{r}\right\|_{L^{2}\left(S^{2}\right)}^{2}+\left\|\nabla_{\omega} U_{r}\right\|_{L^{2}\left(S^{2}\right)}^{2} \mathrm{~d} r<+\infty .
$$


Consequently, by the Schwarz inequality,

$$
\int_{1}^{\infty}\left\|\partial_{r} U_{r}\right\|_{L^{2}\left(S^{2}\right)} \mathrm{d} r<+\infty
$$

which implies the existence of a limit $U$ for $U_{r}$ in $L^{2}\left(S^{2}\right)$. Moreover, in $\mathcal{D}^{\prime}\left(S^{2}\right), \nabla_{\omega} U$ is the limit, as $R$ goes to $\infty$, of

$$
F_{R}=\nabla_{\omega} \int_{R}^{R+1} U_{r} \mathrm{~d} r=\int_{R}^{R+1} \nabla_{\omega} U_{r} \mathrm{~d} r
$$

and

$$
\left\|F_{R}\right\|_{L^{2}\left(S^{2}\right)}^{2} \leqslant \int_{R}^{R+1}\left\|\nabla_{\omega} U_{r}\right\|_{L^{2}\left(S^{2}\right)}^{2} \mathrm{~d} r
$$

which goes to 0 in view of (4.1). This shows that $U$ is a constant $c$.

If $u \in \dot{H}^{1}$, then, by Hardy's inequality,

$$
\int_{0}^{\infty}\left\|U_{r}\right\|_{L^{2}\left(S^{2}\right)}^{2} \mathrm{~d} r=\int_{\mathbb{R}^{3}} \frac{|u(x)|^{2}}{|x|^{2}} \mathrm{~d} x<+\infty
$$

which implies $c(u)=0$. Conversely, if $c(u)=0$, then

$$
U_{r}=-\int_{r}^{\infty} \partial_{\rho} U_{\rho} \mathrm{d} \rho
$$

implies, by the Schwarz inequality,

$$
\left\|U_{r}\right\|_{L^{2}\left(S^{2}\right)}^{2} \leqslant \frac{1}{r}\|\nabla u\|_{L^{2}(|x| \geqslant r)}^{2}=\frac{\mathrm{o}(1)}{r},
$$

as $r$ goes to infinity. Let $\chi \in C_{0}^{\infty}\left(\mathbb{R}^{3}\right)$ be supported in $\{|x| \leqslant 2\}$ with $\chi=1$ for $|x| \leqslant 1$, and let

$$
u_{n}(x)=\chi\left(\frac{x}{n}\right) u(x)
$$

so that $u_{n}$ converges to $u$ in $\mathcal{D}^{\prime}\left(\mathbb{R}^{3}\right)$ as $n$ goes to infinity. We have

$$
\nabla u_{n}(x)-\nabla u(x)=\left(\chi\left(\frac{x}{n}\right)-1\right) \nabla u(x)+\frac{1}{n} u(x) \nabla \chi\left(\frac{x}{n}\right)
$$

from which we infer, as $n \rightarrow \infty$,

$$
\left\|\nabla u_{n}-\nabla u\right\|_{L^{2}}^{2} \leqslant \mathrm{o}(1)+\frac{C}{n^{2}} \int_{n \leqslant|x| \leqslant 2 n}|u(x)|^{2} \mathrm{~d} x \leqslant \mathrm{o}(1)
$$

in view of the above estimate on $\left\|U_{r}\right\|_{L^{2}\left(S^{2}\right)}^{2}$. Using the Sobolev inequality, we conclude that $\left(u_{n}\right)$ is a Cauchy sequence in $L^{6}$, hence $u \in \dot{H}^{1}$. This completes the proof of Lemma 7 .

Remark 4.2. The above proof easily extends to functions $u$ satisfying

$$
\nabla u \in L^{p}\left(\mathbb{R}^{d}\right) \text { with } p<d .
$$

A consequence of this result is the well-known fact that, for every $u$ such that $\nabla u \in L^{p}\left(\mathbb{R}^{d}\right), p<d$, there exists $c \in \mathbb{C}$ such that $u-c \in L^{p^{*}}\left(\mathbb{R}^{3}\right)$, where $p^{*}$ is the Sobolev exponent; see e.g. Theorem 4.5.9 of Hörmander [14] for a different proof. The case $p=2, d=2$ is more problematic, as suggested by the following example,

$$
u(x)=\exp \left(\mathrm{i}[\log (2+|x|)]^{\alpha}\right), \quad \alpha<\frac{1}{2} .
$$


It is now easy to complete the proof of Proposition 4.1. Given $u \in E$, denote by $c(u)$ the constant given by Lemma 1 . Since $|u|^{2}-1 \in L^{2}$, we have

$$
+\infty>\int_{0}^{\infty} r^{2}\left\|\left|U_{r}\right|^{2}-1\right\|_{L^{2}\left(S^{2}\right)}^{2} \mathrm{~d} r \geqslant R^{2} \int_{R}^{R+1}\left\|\left|U_{r}\right|-1\right\|_{L^{2}\left(S^{2}\right)}^{2} \mathrm{~d} r
$$

and the right-hand side is equivalent to $4 \pi R^{2}|| c(u)|-1|^{2}$ as $R \rightarrow \infty$, which imposes $|c(u)|=1$. It remains to set

$$
v=u-c(u)
$$

and to observe that $c(v)=0$, hence $v \in \dot{H}^{1}$, and finally in $F_{c(u)}$.

Let us prove that the distance functions $d_{E}$ and $\delta_{E}$ are equivalent. It is enough to establish that there exists $A>0$ such that, for complex numbers $c, \tilde{c}$ of modulus 1 , for functions $v, \tilde{v}$ in $\dot{H}^{1}\left(\mathbb{R}^{3}\right)$,

$$
\frac{1}{A}\left(|c-\tilde{c}|+\|\nabla v-\nabla \tilde{v}\|_{L^{2}}\right) \leqslant\|c+v-\tilde{c}-\tilde{v}\|_{X^{1}+H^{1}} \leqslant A\left(|c-\tilde{c}|+\|\nabla v-\nabla \tilde{v}\|_{L^{2}}\right) .
$$

If $\chi \in C_{0}^{\infty}\left(\mathbb{R}^{3}\right)$ with $\chi=1$ near the origin, then it is clear that $\chi(D)$ maps $\dot{H}^{1}$ into $X^{1}$, while $1-\chi(D)$ maps $\dot{H}^{1}$ into $H^{1}$. Consequently, writing

$$
c+v-\tilde{c}-\tilde{v}=c-\tilde{c}+\chi(D)(v-\tilde{v})+(1-\chi(D))(v-\tilde{v}),
$$

we obtain the second inequality in (4.2). Conversely, if $u$ belongs to $X^{1}+H^{1}$, then, by Lemma 7 ,

$$
|c(u)|^{2}=\lim _{R \rightarrow \infty} \frac{1}{4 \pi} \int_{R \leqslant|x| \leqslant R+1} \frac{|u(x)|^{2}}{|x|^{2}} \mathrm{~d} x,
$$

which implies

$$
|c(u)| \leqslant A\|u\|_{L^{\infty}+L^{2}} \leqslant A\|u\|_{X^{1}+H^{1}},
$$

and therefore

$$
|c-\tilde{c}| \leqslant A\|c+v-\tilde{c}+\tilde{v}\|_{X^{1}+H^{1}} .
$$

Since, on the other hand, we have trivially

$$
\|\nabla v-\nabla \tilde{v}\|_{L^{2}} \leqslant\|c+v-\tilde{c}-\tilde{v}\|_{X^{1}+H^{1}},
$$

this completes the proof of (4.2).

One advantage of the structure provided by Proposition 4.1 arises when studying how the energy functional measures the distance in $E$ to the circle of constants,

$$
S^{1}=\{u \in E: \mathcal{E}(u)=0\} .
$$

It turns out that the situation is quite different in dimension 2 and dimension 3.

Proposition 4.3. If $d=3$, there exists $A>0$ such that, for every $u \in E$,

$$
A^{-1} d_{E}\left(u, S^{1}\right)^{2} \leqslant \mathcal{E}(u) \leqslant A d_{E}\left(u, S^{1}\right)^{2} .
$$

If $d=2$, there exists a sequence $\left(u_{n}\right)$ in $E$ such that

$$
\mathcal{E}\left(u_{n}\right) \rightarrow 0 \text { but } d\left(u_{n}, S^{1}\right) \geqslant c_{0}>0 .
$$

Proof. First assume $d=3$. In view of the expression of $\delta_{E}$, we have, for every $c \in S^{1}$,

$$
\delta_{E}(u, c)^{2} \simeq|c(u)-c|^{2}+\mathcal{E}(u)
$$


therefore, by Proposition 4.1,

$$
d_{E}\left(u, S^{1}\right)^{2} \simeq \mathcal{E}(u) .
$$

If $d=2$, we consider

$$
u_{n}(x)=\exp \left(\frac{\mathrm{i}}{n} \phi(x)\right), \quad \phi(x)=[\log (2+|x|)]^{\alpha}, \quad 0<\alpha<\frac{1}{2} .
$$

Then it is clear that $\left|u_{n}\right|=1$ and

$$
\left\|\nabla u_{n}\right\|_{L^{2}}=\frac{1}{n}\|\nabla \phi\|_{L^{2}}
$$

so that $\mathcal{E}\left(u_{n}\right)$ tends to 0 . On the other hand, $u_{n}(x)$ tends to 1 uniformly for $x$ in the unit disc $D$, while, uniformly for $x \in y_{n}+D$, with

$$
\left|y_{n}\right|=\mathrm{e}^{(\theta n)^{1 / \alpha}}, \quad \theta>0
$$

we have

$$
u_{n}(x) \rightarrow \mathrm{e}^{\mathrm{i} \theta} .
$$

Since

$$
d_{E}(f, g) \geqslant\|f-g\|_{X^{1}+H^{1}} \geqslant \sup _{y \in \mathbb{R}^{2}}\|f-g\|_{L^{2}(y+D)}
$$

we conclude that $d_{E}\left(u_{n}, S^{1}\right)$ stays away from 0.

\subsection{Description of the dynamics}

It is now easy to describe the dynamics of (1.1) in terms of the decomposition provided by Proposition 4.1.

Proposition 4.4. Let $u_{0}=c+v_{0}$ with $c$ of modulus 1 and $v_{0} \in F_{c}$. Then the solution $u \in C(\mathbb{R}, E)$ of $(1.1)$ is given by

$$
u(t)=c+v(t)
$$

where $v \in C\left(\mathbb{R}, F_{c}\right)$ is the unique solution of

$$
\mathrm{i} \partial_{t} v+\Delta v=\left(|v|^{2}+2 \operatorname{Re}\left(c^{-1} v\right)\right) v .
$$

Proof. For every $t$, we decompose

$$
u(t)=c(t)+v(t)
$$

with $c \in C(\mathbb{R}, \mathbb{C})$ and $v \in C\left(\mathbb{R}, \dot{H}^{1}\right)$. Proposition 4.3 then reduces to the fact that $c(t)=c$ for every $t$. For every $t$, observe that, by Theorem 1.1 and Proposition 2.3,

$$
u(t)=u_{L}(t)+H^{1}=u_{0}+H^{1}=c+\dot{H}^{1} .
$$

This completes the proof.

Remark 4.5. Of course it is possible to solve directly Eq. (4.3) by using Strichartz estimates on the gradient, providing a slightly different proof of Theorem 1.1 in the special case $d=3$.

\section{The four-dimensional case}

In this last part, we sketch the proof of the following generalization of Theorem 1.1 to the four-dimensional case. Since the cubic nonlinearity is critical, existence is only obtained for small energy data. 
Theorem 5.1. Assume $d=4$. There exists $\delta>0$ such that, for every $u_{0} \in E$ such that $\mathcal{E}\left(u_{0}\right) \leqslant \delta$, there exists a unique solution of $(1.1) u \in C(\mathbb{R}, E)$ with $\left.\nabla u \in L_{\text {loc }}^{2}(\mathbb{R}), L^{4}\left(\mathbb{R}^{4}\right)\right)$ and $u(0)=u_{0}$. Moreover, the energy of $u$ is constant, and the flow map such defined enjoys the properties of regularity and Lipschitz continuity stated in Theorem 1.1 and in Proposition 4.4.

Proof. According to Remark 4.2 and Proposition 4.4, we shall look $u$ under the form

$$
u(t)=c+v(t),
$$

where $c \in S^{1}$ and $v(t)$ belongs to

$$
F_{c}=\left\{v \in \dot{H}^{1}\left(\mathbb{R}^{4}\right): c+v \in E\right\} .
$$

The specificity of dimension 4 is that the space $F_{c}$ is a (real) vector space. Indeed, because of the Sobolev inclusion $\dot{H}^{1}\left(\mathbb{R}^{4}\right) \subset L^{4}\left(\mathbb{R}^{4}\right)$, we have

$$
F_{c}=\left\{v \in \dot{H}^{1}\left(\mathbb{R}^{4}\right): \operatorname{Re}\left(c^{-1} v\right) \in L^{2}\left(\mathbb{R}^{4}\right)\right\}
$$

and, if $\mathcal{E}(c+v)$ is small enough,

$$
\mathcal{E}(c+v) \simeq\|\nabla v\|_{L^{2}}^{2}+\left\|\operatorname{Re}\left(c^{-1} v\right)\right\|_{L^{2}}^{2} .
$$

In particular, the approximation Lemma 6 still holds, though the proof we gave in Section 3 breaks down if $d=4$. The main argument for local existence is the following estimate of the Duhamel term (3.4), which is a consequence of the nonhomogeneous Strichartz estimates (3.3),

$$
\begin{aligned}
& \sup _{|t| \leqslant T}\|\Phi(c+v)(t)\|_{H^{1}}+\|\nabla \Phi(c+v)\|_{L_{T}^{2}\left(L^{4}\right)} \\
& \quad \leqslant C\left(T \sup _{|t| \leqslant T} \sqrt{\mathcal{E}(c+v(t))}+\left(\sqrt{T}+\|\nabla v\|_{L_{T}^{2}\left(L^{4}\right)}\right) \sup _{|t| \leqslant T} \mathcal{E}(c+v(t))\right) .
\end{aligned}
$$

If $\mathcal{E}\left(c+v_{0}\right) \leqslant \delta$, it is then possible to solve the Duhamel equation

$$
v(t)=S(t)\left(v_{0}\right)+\Phi(c+v)(t)
$$

by a contraction argument in the space

$$
\begin{gathered}
Y_{T, \delta}=\left\{v \in C\left([-T, T], F_{c}\right): \nabla v \in L^{2}\left([-T, T], L^{4}\left(\mathbb{R}^{4}\right)\right),\right. \\
\left.\sup _{|t| \leqslant T} \mathcal{E}(c+v(t)) \leqslant A \delta,\|\nabla v\|_{L_{T}^{2}\left(L^{4}\right)} \leqslant B \sqrt{\delta}\right\}
\end{gathered}
$$

for $\delta$ and $T$ small enough.

\section{Acknowledgements}

I am grateful to J.-C. Saut and to D. Smets for having drawn my attention to this problem. I wish to thank C. Gallo for a careful reading of the manuscript and a remark which led me to a simpler proof of Propositions 2.3 and 4.4.

\section{References}

[1] F. Béthuel, G. Orlandi, D. Smets, Vortex rings for the Gross-Pitaevskii equation, J. Eur. Math. Soc. 6 (2004) 17-94.

[2] F. Béthuel, J.C. Saut, Travelling waves for the Gross-Pitaevskii equation I, Ann. Inst. H. Poincaré Phys. Théor. 70 (1999) $147-238$.

[3] H. Brezis, T. Gallouët, Nonlinear Schrödinger evolution equations, Nonlinear Anal. 4 (1980) 677-681.

[4] T. Cazenave, Semilinear Schrödinger Equations, Courant Lecture Notes in Math., vol. 10, New York University, American Mathematical Society, Providence, RI, 2003.

[5] T. Frisch, Y. Pomeau, S. Rica, Transition to dissipation in a model of superflow, Phys. Rev. Lett. 69 (1992) 1644-1647.

[6] C. Gallo, Schrödinger group on Zhidkov spaces, Adv. Differential Equations 9 (2004) 509-538.

[7] J. Ginibre, G. Velo, On a class of nonlinear Schrödinger equations, J. Funct. Anal. 32 (1979) 1-71.

[8] J. Ginibre, G. Velo, The global Cauchy problem for the nonlinear Schrödinger equation, Ann. Inst. H. Poincaré Anal. Non Linéaire 2 (1985) 309-327. 
[9] O. Goubet, Two remarks on solutions of Gross-Pitaevskii equations on Zhidkov spaces, Preprint, 2005.

[10] P. Gravejat, A non-existence result for supersonic travelling waves in the Gross-Pitaevskii equation, Comm. Math. Phys. 243 (2003) 93-103.

[11] P. Gravejat, Decay of travelling waves in the Gross-Pitaevskii equation, Ann. Inst. H. Poincaré Anal. Non Linéaire 21 (2004) $591-637$.

[12] P. Gravejat, Limit at infinity and non-existence result for sonic travelling waves in the Gross-Pitaevskii equation, Differential Integral Equations 17 (2004) 1213-1232.

[13] E.P. Gross, J. Math. Phys. 4 (1963) 195.

[14] L. Hörmander, The Analysis of Linear Partial Differential Operators, vol. 1, Springer-Verlag, 1983.

[15] T. Kato, On nonlinear Schrödinger equations, Ann. Inst. H. Poincaré Phys. Théor. 46 (1987) 113-129.

[16] M. Keel, T. Tao, Endpoint Strichartz estimates, Amer. J. Math. 120 (1998) 955-980.

[17] C. Nore, M. Abid, M. Brachet, Decaying Kolmogorov turbulence in a model of superflow, Phys. Fluids 9 (1997) $2644-2669$.

[18] L.P. Pitaevskii, Sov. Phys. JETP 13 (1961) 451.

[19] C. Sulem, P.L. Sulem, The Nonlinear Schrödinger Equation. Self-Focusing and Wave Collapse, Appl. Math. Sci., vol. 139, Springer-Verlag, 1999.

[20] P.E. Zhidkov, The Cauchy problem for a nonlinear Schrödinger equation, Dubna, 1987.

[21] P.E. Zhidkov, Korteweg-de Vries and Nonlinear Schrödinger Equations: Qualitative Theory, Lecture Notes in Math., vol. 1756, SpringerVerlag, 2001. 